\section{Efecto del estado nutricional neonatal en el riesgo de síndrome metabólico en niños obesos de 2 comunas de la Región del Bío-Bío}

\author{
JORGE SAPUNAR ${ }^{1, \mathrm{a}}$, PAULINA BUSTOS ${ }^{2, \mathrm{a}}$, KATIA SÁEZ ${ }^{3, \mathrm{~b}}$, \\ SERGIO MUÑOZ ${ }^{1, \mathrm{~b}}$, SYLVIA ASENJO $^{4}$
}

\section{Effects of neonatal nutritional status on the risk for metabolic syndrome in Chilean obese children}

Background: Neonatal malnutrition defined by birth weight $(B W)$ is a risk factor for obesity and cardio-metabolic diseases in adults. Neonatal ponderal index (NPI) may have better diagnostic value than BW to establish nutritional status. Aim: To determine the effect of neonatal nutritional status, established by the three NPI curves available in Chile, on the risk of Metabolic Syndrome (MS) in obese school children. Material and Methods: A nested case/control study in a sample of 410 obese school children aged 10 to 16 years (57\% males) was performed. The dichotomous response variable was the presence of MS defined as International Diabetes Federation (IDF) or Cook's criteria. The exposure variable was having NPI < percentile ( $p)$ 10. Results: The frequency of MS was 36 and 39\% according to the IDF and Cook criteria, respectively. The proportion of children with neonatal malnutrition exceeded 20\%. A significantly increased risk for MS was only found when PNI was defined according to Lagos's Table and MS was defined using IDF criteria. Having a PNI > p90, however, showed a trend towards a reduced risk of MS, which only reached significance using Lagos's Table and Cook's Criteria. Conclusions: Neonatal malnutrition defined by NPI is common in obese school children. The condition of neonatal under nutrition defined as PNI < p10 may be a risk factor for developing MS. Instead, having a NPI > p90 could be protective.

(Rev Med Chile 2014; 142: 1523-1529)

Key words: Fetal Development; Infant Nutrition Disorders; Metabolic Syndrome X.

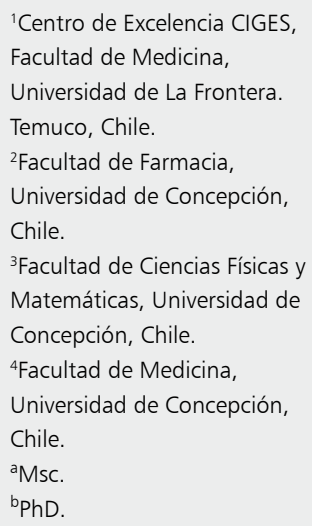

I a obesidad común es una de las enfermedades crónicas más frecuentes en población infanto-juvenil, tanto a nivel mundial ${ }^{1}$ como en Chile ${ }^{2,3}$. La Región del Bío-Bío tiene una alta prevalencia de sobrepeso y obesidad en niños $\mathrm{y}$ adolescentes, que alcanza mayor importancia en las edades extremas de este segmento de la población ${ }^{4}$.

Los trastornos nutricionales por exceso en escolares se han asociado con anomalías metabólicas y endocrinas como hiperinsulinismo, intolerancia a la glucosa y dislipidemia que, junto a la hipertensión arterial, conducen tempranamente a la aparición de enfermedad cardiovascular y diabetes mellitus ${ }^{5,6}$. El conjunto de estos factores de riesgo constituiría el síndrome metabólico (SM), condición definida para población adulta por el National Cholesterol Education Program 
Adults Treatment Panel (ATP III) ${ }^{7}$. En pediatría, la primera definición operacional de SM fue una modificación de la definición ATP III, propuesta en el estudio de $\mathrm{Cook}^{8}$. Posteriormente, la Internacional Diabetes Federation (IDF) propuso una nueva definición de SM para escolares mayores de 10 años ${ }^{9}$. Múltiples estudios han buscado conocer la frecuencia del SM ${ }^{10-16}$; en Chile, nuestro grupo comunicó que 37,6\% de una muestra de escolares con obesidad tenía SM mediante el criterio IDF $^{17}$; posteriormente, Eyzaguirre y cols. ${ }^{18}$ encontraron $22,7 \%$ de SM mediante el criterio de Cook y $45 \%$ mediante el criterio de Ferranti ${ }^{19}$.

La desnutrición neonatal es considerada un factor de riesgo para obesidad y enfermedades cardiometabólicas en el adulto ${ }^{20,21}$. Para la valoración neonatal del estado nutricional se ha utilizado la relación peso de nacimiento y edad gestacional $^{22-24}$, sin embargo, podría tener mejor valor diagnóstico la estimación del índice ponderal neonatal (IPN) ${ }^{25,26}$.

La función que asocia el peso de nacimiento y la glicemia post carga en escolares asume forma de $U$, teniendo los niños que fueron pequeños y grandes para la edad gestacional menor sensibilidad a la insulina que sus controles normales ${ }^{27-29}$. Sin embargo, al evaluar el riesgo de síndrome metabólico infanto-juvenil de acuerdo al estado nutricional neonatal, los resultados han sido contrapuestos $^{30,31}$.

El propósito del presente estudio es conocer el efecto del estado nutricional neonatal, establecido por las 3 curvas de IPN disponibles en Chile, en el riesgo de SM, definido por los criterios de la IDF y de Cook, en escolares obesos de las comunas de Concepción y Coronel.

\section{Material y Método}

Se realizó un estudio de casos y controles anidado en una cohorte de 410 escolares con obesidad de las comunas de Concepción y Coronel, Región del Bío-Bío, Chile, que participaron en un estudio de prevalencia de síndrome metabólico ${ }^{17}$. La cohorte la conformaban escolares de 10 a 16 años de edad, 233/410 eran varones (56,8\%).

La condición de obeso se estableció de acuerdo al $z$ score del índice de masa corporal (IMC) corregidos por edad y sexo definido en las Tablas de Centers for Disease Control and Prevention $(\mathrm{CDC})^{32}$.
La variable de respuesta dicotómica fue la condición de síndrome metabólico (SM) según el criterio de la IDF9 y el propuesto por Cook $^{8}$. La obtención de los parámetros para establecer el diagnóstico de síndrome metabólico (presión arterial, cintura, niveles séricos en ayuno de glucosa, colesterol HDL y triglicéridos) se describe previamente ${ }^{17}$.

La variable de exposición dicotómica fue la condición de desnutrición neonatal definida como IPN $<$ p 10 según las curvas propuestas por Juez ${ }^{33}$, por la rama de neonatología de la Sociedad Chilena de Pediatría (Pittaluga/Alarcón) ${ }^{34}$ y por Lagos ${ }^{35}$. El IPN se calculó de acuerdo a la fórmula ${ }^{25,26}$ :

$$
100 \times \text { (peso en } \mathrm{g}) /(\text { talla en } \mathrm{cm})^{3}
$$

La edad gestacional, peso y talla de nacimiento se obtuvieron desde los registros de atención del parto.

Para evaluar la asociación entre las condiciones de desnutrición neonatal y de síndrome metabólico se consideraron como variables de control la edad y el $z$ score de IMC al momento de ingresar a la cohorte, el género, los antecedentes parentales de diabetes mellitus 2 (DM2) y obesidad.

Para el análisis de los datos se utilizó el programa estadístico STATA 11 (STATA Corp.). Se describe la frecuencia de SM de la cohorte según IDF y Cook, la frecuencia de IPN $<$ p 10 y $>$ p90 según Juez, Pittaluga/Alarcón y Lagos. Se compara la frecuencia de variables de control según condición de SM por IDF y Cook. Se estima el riesgo de SM por IDF y Cook de los niños obesos de la cohorte como OR mediante análisis univariado y multivariado. Finalmente, se evalúa la tendencia del riesgo de SM considerando el estado nutricional neonatal, expresado como peso de nacimiento (PN) e IPN corregidos por la edad gestacional (PN x 40/Edad Gestacional en semanas; IPN x 40/Edad Gestacional en semanas) como variables continuas.

El estudio de la cohorte fue aprobado por el Comité de Bioética de la Universidad de Concepción ${ }^{17}$.

\section{Resultados}

La frecuencia de síndrome metabólico en la cohorte de niños obesos fue $36 \%$ según el criterio IDF y $39 \%$ por el criterio de Cook $(p=0,8125)$. 


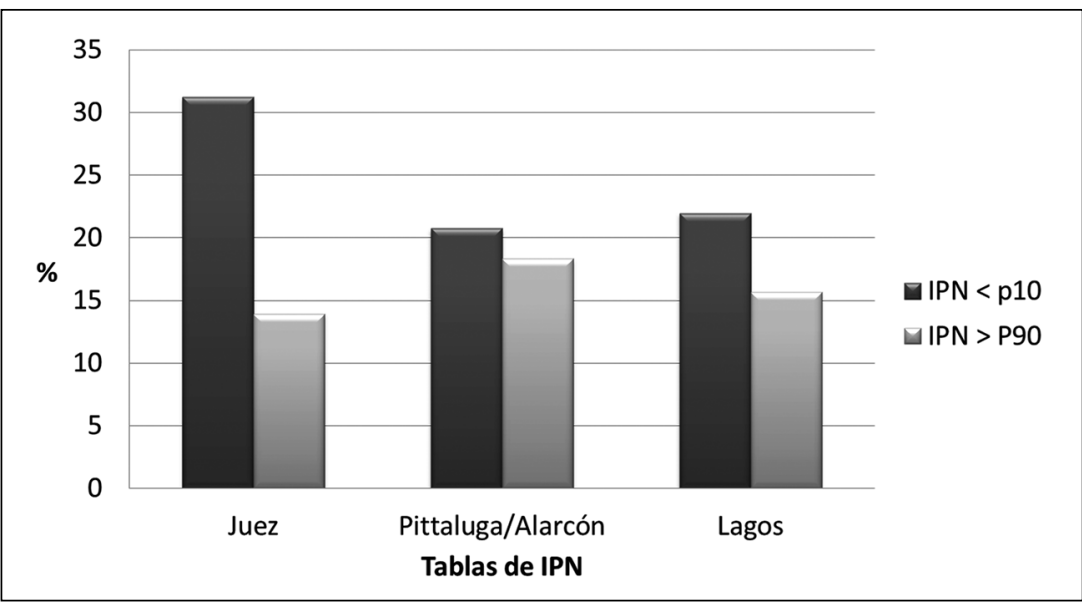

Figura 1. Frecuencia de trastornos nutricionales neonatales en 410 niños obesos de las Comunas de Concepción y Coronel según curva de IPN.
En la Figura 1 se puede apreciar la frecuencia de trastornos nutricionales neonatales (IPN $<$ p10 y $>$ p90). La proporción de sujetos con desnutrición neonatal varió de acuerdo a la curva utilizada (Juez, Pittaluga/Alarcón, Lagos), pero en todos los casos superó $20 \%$.
Al comparar la distribución y magnitud de las variables de exposición y control (IPN $<$ p 10 para cada curva, valor de IPN, IMC actual y edad) entre los casos (SM) y controles (Sin SM) sólo fueron diferentes la edad e IMC actual con el criterio IDF y sólo el IMC con el criterio de Cook (Tabla 1).

\section{Tabla 1. Variables de exposición y control según condición de síndrome metabólico en 410 niños obesos de las Comunas de Concepción y Coronel, clasificados de acuerdo a la condición de SM de acuerdo a IDF y Cook}

\begin{tabular}{|c|c|c|c|}
\hline Variable & $\begin{array}{c}\text { SM (+) IDF } \\
n=149\end{array}$ & $\begin{array}{c}\text { SM (-) IDF } \\
n=261\end{array}$ & Valor $\mathbf{p}$ \\
\hline Edad (IC 95\%) & $14,90(2,20)$ & $13,24(2,26)$ & $p<0,001$ \\
\hline$\%$ varones & 56,4 & 57,1 & NS \\
\hline IMC (IC 95\%) & $34,21(4,41)$ & $31,07(3,67)$ & $p<0,001$ \\
\hline IPN (IC 95\%) & $2,68(0,37)$ & $2,69(0,44)$ & NS \\
\hline$\%$ IPN < p10 (Juez) & 32,21 & 30,65 & NS \\
\hline \% IPN < p10 (Pittaluga/Alarcón) & 20,13 & 21,07 & NS \\
\hline$\%$ IPN < p10 (Lagos) & 21,40 & 22,22 & NS \\
\hline Variable & $\begin{array}{c}\text { SM }(+) \text { Cook } \\
n=159\end{array}$ & $\begin{array}{c}\text { SM (-) Cook } \\
n=251\end{array}$ & Valor $p$ \\
\hline Edad (IC 95\%) & $13,88(2,30)$ & $13,85(2,43)$ & NS \\
\hline$\%$ varones & 56,4 & 49,0 & NS \\
\hline IMC (IC 95) & $33,42(4,61)$ & $31,45(3,78)$ & $\mathrm{p}<0,001$ \\
\hline IPN (IC 95\%) & $2,69(0,39)$ & $2,68(0,40)$ & NS \\
\hline$\%$ IPN < p10 (Juez) & 30,8 & 31,4 & NS \\
\hline \% IPN < p10 (Pittaluga/Alarcón) & 18,86 & 21,9 & NS \\
\hline$\%$ IPN < p10 (Lagos) & 20,1 & 23,1 & NS \\
\hline
\end{tabular}


Considerando los múltiples factores que podrían afectar la ocurrencia del síndrome metabólico, quisimos conocer el efecto de la desnutrición neonatal en el riesgo de síndrome metabólico en un modelo de regresión logística múltiple que incluyó como variables de control género, edad, $\mathrm{z}$ score de IMC, antecedentes parentales de DM2 y obesidad.

En la Tabla 2 se presenta el análisis multivariado del riesgo de síndrome metabólico para la curva de IPN de Juez. En este modelo sólo tienen efecto significativo en el riesgo de SM el $z$ score del IMC actual con OR de 7,29 para el criterio IDF (valor $\mathrm{p}=0,004)$ y 13,63 para el criterio de Cook (valor $\mathrm{p}<0,001)$. El efecto de la desnutrición neonatal no alcanzó significación estadística. En la Tabla 3 se presenta el análisis multivariado del riesgo de síndrome metabólico para la curva de IPN de Pittaluga/Alarcón. En este caso, nuevamente sólo tiene efecto el $z$ score del IMC. En la Tabla 4 se presenta el análisis multivariado del riesgo de síndrome metabólico para la curva de IPN de Lagos. En este caso la desnutrición neonatal se asoció con un aumento en el riesgo de SM según el criterio IDF (OR 2,12 valor $\mathrm{p}=0,047$ ).

Considerando los hallazgos de Eyzaguirre y cols. ${ }^{31}$, a continuación evaluamos el efecto de tener un IPN > p 90 en el riesgo de SM (Tabla 5). En ella se puede apreciar una tendencia a la reducción de riesgo de SM, que sólo alcanzó significación con la curva de Lagos para SM según Cook.

$\mathrm{Al}$ estratificar el valor de IPN corregido por edad gestacional y compararlo con el logaritmo de la OR de SM según IDF y Cook no se aprecia asociación. La distribución de los puntos no asume una forma definida. Al estratificar el valor de PN corregido por edad gestacional y compararlo con el logaritmo de la OR de SM según IDF y Cook,

Tabla 2. Riesgo de síndrome metabólico e IPN < p10 por curva de Juez. Análisis multivariado

\begin{tabular}{|lcccc|}
\hline Variables & OR & SM IDF & \multicolumn{2}{c|}{ SM Cook } \\
IPN $<10$ & 1,74 & Valor $\mathbf{p}$ & OR & Valor $\mathbf{p}$ \\
Sexo & 1,05 & 0,099 & 1,26 & 0,459 \\
IMC z score & 7,29 & 0,877 & 1,08 & 0,794 \\
DM madre & 1,42 & 0,004 & 13,63 & 0,000 \\
DM padre & 0,72 & 0,308 & 1,53 & 0,721 \\
Obesidad madre & 0,77 & 0,623 & 1,13 & 0,348 \\
Obesidad padre & 1,31 & 0,420 & 0,71 & 0,257 \\
\hline
\end{tabular}

Tabla 3. Riesgo de síndrome metabólico e IPN < p10 por curva de Pittaluga/Alarcón. Análisis multivariado

\begin{tabular}{|c|c|c|c|c|}
\hline \multirow{2}{*}{ Variables } & \multicolumn{2}{|c|}{ SM IDF } & \multicolumn{2}{|c|}{ SM Cook } \\
\hline & OR & Valor $p$ & OR & Valor $p$ \\
\hline IPN $<10$ & 1,94 & 0,087 & 1,52 & 0,257 \\
\hline Sexo & 1,09 & 0,782 & 1,10 & 0,737 \\
\hline IMC z score & 7,49 & 0,004 & 13,90 & 0,000 \\
\hline DM madre & 1,43 & 0,372 & 1,54 & 0,256 \\
\hline DM padre & 0,72 & 0,613 & 1,14 & 0,826 \\
\hline Obesidad madre & 0,76 & 0,408 & 0,69 & 0,236 \\
\hline Obesidad padre & 1,34 & 0,366 & 0,86 & 0,639 \\
\hline
\end{tabular}


Tabla 4. Riesgo de síndrome metabólico e IPN < p10 por curva de Lagos. Análisis multivariado

\begin{tabular}{|lcccc|}
\hline Variables & OR & SM IDF & \multicolumn{2}{c|}{ SM Cook } \\
IPN $<10$ & 2,12 & Valor $\mathbf{p}$ & OR & Valor $\mathbf{p}$ \\
Sexo & 1,06 & 0,047 & 1,68 & 0,144 \\
IMC z score & 7,27 & 0,859 & 1,08 & 0,785 \\
DM madre & 1,43 & 0,004 & 13,86 & 0,000 \\
DM padre & 0,71 & 0,373 & 1,55 & 0,255 \\
Obesidad madre & 0,74 & 0,612 & 1,14 & 0,817 \\
Obesidad padre & 1,31 & 0,370 & 0,68 & 0,217 \\
\hline
\end{tabular}

Tabla 5. Riesgo de síndrome metabólico e IPN > p 90 según las curva de Juez, Pittaluga/Alarcón y Lagos

\begin{tabular}{|lcccc|}
\hline Variables & \multicolumn{2}{c}{ SM IDF } & \multicolumn{2}{c|}{ SM Cook } \\
\hline IPN > p90 Juez & OR & Valor $\mathbf{p}$ & OR & Valor $\mathbf{p}$ \\
IPN > p90 Pittaluga-Alarcón & 0,73 & 0,428 & 0,48 & 0,06 \\
IPN > p90 Lagos & 0,84 & 0,641 & 0,57 & 0,104 \\
\hline
\end{tabular}

se aprecia una distribución en U con mayor riesgo de SM según IDF en los PN corregidos por edad gestacional extremos.

\section{Discusión}

Nuestro estudio evaluó por primera vez la asociación entre el estado nutricional neonatal expresado como IPN y el riesgo de SM de escolares con obesidad. Previamente se había estudiado el efecto del peso del recién nacido en la frecuencia de los componentes del SM con resultados discordantes $^{30,31}$.

El estudio de la asociación entre el estado nutricional neonatal y desenlaces metabólicos en la etapa infanto-juvenil se ve dificultado por el efecto de otras variables, como el estado nutricional actual y porque la función que describe tal asociación tiene forma de $\mathrm{U}$, donde los recién nacidos pequeños y grandes para edad gestacional tienen posteriormente resistencia a la insulina ${ }^{27,29}$. Otro aspecto que afecta en análisis es la controversia en torno a la definición más apropiada de SM en el período infanto-juvenil ${ }^{8,9}$.
En homología al índice de masa corporal (IMC), el IPN busca expresar mejor el estado nutricional neonatal que el peso de nacimiento al considerar la talla de nacimiento ${ }^{25,26}$. Por ello en nuestro país se han propuesto varias tablas que definen la normalidad entre los percentiles 10 y $90^{33-35}$.

En nuestra muestra de escolares con obesidad de la Región del Bío-Bío, la frecuencia de SM fue algo mayor que la encontrada por Eyzaguirre en niños de la Región Metropolitana, utilizando el mismo criterio diagnóstico ${ }^{18}$. Independientemente de la tabla utilizada, la frecuencia de niños obesos que tuvieron desnutrición neonatal (IPN $<$ p10) fue superior a $20 \%$.

Al evaluar el efecto de la condición de desnutrición neonatal en el riesgo de SM mediante regresión logística múltiple, considerando variables de control, sólo encontramos aumento significativo del riesgo al utilizar la tabla de Lagos para $\operatorname{IPN}^{35} \mathrm{y}$ el criterio para SM de IDF9. La falta de asociación entre SM y la condición de desnutrición neonatal establecida por las tablas de Juez y Pittaluga/ Alarcón se podría atribuir a que el percentil 10 de IPN no sea el mejor valor de corte para predecir 
Malnutrición neonatal como riesgo de síndrome metabólico - J. Sapunar et al

riesgo de SM. Adicionalmente, la muestra de población utilizada por Lagos para construir su tabla ${ }^{35}$ podría asemejarse más a nuestra muestra de escolares que las utilizadas en las tablas de Juez y Pittaluga/Alarcón ${ }^{33,34}$.

El resultado más sorprendente de nuestro estudio fue la reducción en el riesgo de SM observada en escolares obesos que tuvieron IPN $>90$, hallazgo que contrasta con la menor sensibilidad a la insulina descrita para este grupo de $\mathrm{RN}$ cuando fueron clasificados en base al peso de nacimiento ${ }^{27-29}$. Adicionalmente, las curvas obtenidas al graficar la relación entre el logaritmo del riesgo de SM y el estado nutricional neonatal fueron diferentes si éste fue descrito por IPN o por el peso de nacimiento corregidos por edad gestacional.

Las Tablas de IPN disponibles en nuestro país incluyen sólo 3 percentiles (10, 50 y 90), lo que no nos permitió explorar otros valores de corte para el estado nutricional neonatal por este criterio y tampoco elaborar curvas para describir la relación entre el riesgo de SM y el percentil de IPN. Para solucionar esta limitación utilizamos el IPN corregido por edad gestacional. Finalmente nuestro estudio fue un análisis post hoc de una cohorte, por lo que el tamaño muestral fue estimado para el estudio original.

En conclusión la desnutrición neonatal definida por IPN es frecuente entre escolares con obesidad. Aunque la variable con la mayor magnitud de efecto en el riesgo metabólico infanto-juvenil es el IMC actual, la condición de desnutrición neonatal definida como IPN $<$ p10 parece ser un factor de riesgo para desarrollar SM en escolares obesos. Las discordancias observadas entre el PN e IPN elevados en relación al riesgo de SM requieren de estudio adicional para establecer si los niños grandes para la edad gestacional realmente constituyen un grupo de riesgo para SM.

\section{Referencias}

1. Berenson GS. Obesity a critical issue in preventive cardiology: the Bogalusa Heart Study. Prev Cardiol 2005; 8: 234-41.

2. Albala C, Vio F, Kain J, Uauy R. Nutrition transition in Latin America: the case of Chile. Nutr Rev 2001; 59: 1706.

3. Albala C, Vio F, Kain J, Uauy YR. Nutrition transition in Chile: determinants and consequences. Public Health
Nutr 2002; 5: 123-8.

4. Ulloa N, Sapunar J, Bustos P, Sáez K, Asenjo S, Taibo M, Cornejo A. [Frequency of obesity and overweight among school age children living in southern Chile]. Rev Med Chile 2010; 138: 1365-72.

5. Sinha R, Fisch G, Teague B, Tamborlane WV, Banyas B, Allen $\mathrm{K}$, et al. Prevalence of impaired glucose tolerance among children and adolescentswith marked obesity. N Engl J Med 2002; 346: 802-10.

6. Aggoun Y. Obesity, metabolic syndrome, and cardiovascular disease. Pediatr Res 2007; 61: 653-9.

7. Expert Panel on Detection, Evaluation, and Treatment of High Blood Cholesterol in Adults (Adult Treatment Panel III). Executive Summary of Third Report of The National Cholesterol Education Program (NCEP). JAMA 200; 285: 2486-97.

8. Cook S, Weitzman M, Auinger P, Nguyen M, Dietz WH. Prevalence of a metabolic syndrome phenotype in adolescents: findings from the third National Health and Nutrition Examination Survey 1988-1994. Arch Pediatr Adolesc Med 2003; 157: 821-7.

9. Zimmet P, Alberti KG, Kaufman F, Tajima N, Silink M, Arslanian S, et al. IDF Consensus Group. The metabolic syndrome in children and adolescents-an IDF consensus report. Pediatr Diabetes 2007; 8: 299-306.

10. Cook S, Auinger P, Li C, Ford ES. Metabolic syndrome rates in United States adolescents, from the National Health and Nutrition Examination Survey, 1999-2002. J Pediatr 2008; 152: 165-70.

11. Sen Y, Kandemir N, Alikasifoglu A, Gonc N, Ozon A. Prevalence and risk factors of metabolic syndrome in obese children and adolescents: the roleof the severity of obesity. Eur J Pediatr 2008; 167: 1183-9.

12. López-Capape M, Alonso M, Colino E, Mustieles C, Corbatón J, Barrio R. Frequency of the metabolic syndrome in obese Spanish pediatric population. Eur J Endocrinol 2006; 155: 313-9.

13. Pajuelo J, Bernui I, Nolberto V, Peña A, Zevillanos L. Síndrome metabólico en adolescentes con sobrepeso y obesidad. An Fac Med Lima 2007; 68: 143-9.

14. Costa RF, Santos NS, Goldraich NP, Barski TF, de Andrade KS, Kruel LFM. Metabolic syndrome in obese adolescents: a comparison of three different diagnostic criteria. J Pediatr (Rio J). 2012; 88: 303-9.

15. Chen F, Wang Y, Shan X, Cheng H, Hou D, Zhao X, et al. Association between Childhood Obesity and MetabolicSyndrome: Evidence from a Large Sample of Chinese Children and Adolescents. PLoS ONE. 2012; 7: e47380.

16. van Vliet M, Heymans MW, von Rosenstiel IA, Brandjes DPM, Beijnen JH. Diamant M. Cardiometabolic risk variables in overweight andobese children: a worldwide 
comparison. Cardiovascular Diabetology 2011; 10: 10615.

17. Bustos P, Sáez K, Gleisner A, Ulloa N, Calvo C, Asenjo S. Metabolic syndrome in obese adolescents. Pediatr Diabetes. 2010; 11: 55-60.

18. Eyzaguirre F, Silva R, Román R, Palacio A, Cosentino $\mathrm{M}$, Vega $\mathrm{V}$, et al. [Prevalence of metabolic syndrome in children and adolescents who consult with obesity]. Rev Med Chile 2011; 139: 732-8.

19. De Ferranti SD, Gauvreau K, Ludwig DS, Neufeld EJ, Newburger JW, Rifai N. Prevalence of the metabolic syndrome in American adolescents: findings from theThird National Health and Nutrition Examination Survey. Circulation 2004; 110: 2494-7.

20. Barker DJP, Osmond C, Golding J, Kuh D, Wadsworth MEJ. Growth in utero, blood pressure in childhood and adult life, and mortality from cardiovascular disease. BMJ 1989; 298: 564-7.

21. Godfrey KM, Barker DJP. Fetal nutrition and adult disease. Am J Clin Nut 2000; 71: 1344S-1352S.

22. Battaglia FC, Lubchenco LO. A practical classification of newborn infants by weight and gestational age. J Pediatr 1967; 71: 159-63.

23. Gruenwald P. Growth of the human fetus. I. Normal growth and its variation. Am J Obstet Gynec 1966; 94: 1112-18.

24. Hadlock FP, Harrist RB, Martínez-Poyer J. In utero analysis of growth: A sonographic weight standard. Radiology 1991; 181: 129-33.

25. Vintzileos AM, Lodeiro JC, Feinstein SJ, Campbell WA, Weinbaum PJ, Nochimson DJ. Value of fetal ponderal index in predicting growth retardation. Obstet Gynecol 1986; 67: 584-8.

26. Yagel S, Zacut D, Igelstein S, Palti Z, Hurwitz A, Rosenn B. In utero ponderal index as a prognostic factor in the evaluation of intrauterine growth retardation. Am J Obstet Gynecol 1987; 157: 415-9.
27. Dabelea D, Pettitt DJ, Hanson RL, Imperatore G, Bennett $\mathrm{PH}$, Knowler WC. Birth weight, type 2 diabetes, and insulin resistance in Pima Indian children and young adults. Diabetes Care 1999; 22: 944-50.

28. Veening MA, Van Weissenbruch MM, Delemarre-Van De Waal HA. Glucose tolerance, insulin sensitivity, and insulin secretion inchildren born small for gestational age. J Clin Endocrinol Metab 2002; 87: 4657-61.

29. Martínez-Aguayo A, Capurro T, Peña V, Iñiguez G, Hernández MI, Ávila A, et al. Comparison of leptin levels, body composition and insulin sensitivity and secretion by OGTT in healthy, early pubertal girls born at either appropriate- or small-for-gestational age. Clin Endocrinol (Oxf). 2007; 67: 526-32.

30. Salonen MK, Kajantie E, Osmond C, Forsén T, Ylihärsilä $\mathrm{H}$, Paile-Hyvärinen $\mathrm{M}$, et al. Role of childhood growth on the risk of metabolic syndrome in obese men and women. Diabetes Metab 2009; 35: 94-100.

31. Eyzaguirre F, Bancalari R, Román R, Silva R, Youlton $\mathrm{R}$, Urquidi $\mathrm{C}$, et al. Prevalence of components of the metabolic syndrome according to birthweight among overweight and obese children and adolescents. J Pediatr Endocrinol Metab 2012; 25: 51-6.

32. http://www.cdc.gov/nccdphp/dnpa/bmi/bmi-forage. htm. Centers for Disease Control and Prevention: BMI for children and teens. April 2003.

33. Juez G, Lucero E, Ventura-Juncá P, González H, Tapia JL, Winter A. Crecimiento intrauterino en recién nacidos chilenos de clase media. Rev Chil Pediatr 1989; 60: 198202.

34. Milad M, Novoa JM, Fabres J, Samame MM, Aspillaga C. Recomendación sobre Curvas de Crecimiento Intrauterino. Rev Chil Pediatr 2010; 81: 264-74.

35. Lagos R, Bustos L, Orellana JJ. Evaluación neonatal del crecimiento intrauterino de recién nacidos en Hospital Regional de Temuco. Comparación con tres estándares nacionales. Rev Chil Obstet Ginecol 2009; 74: 209-16. 